\title{
On the Cayley isomorphism problem for Cayley objects of nilpotent groups of some orders
}

\author{
Edward Dobson \\ Department of Mathematics and Statistics \\ Mississippi State University \\ PO Drawer MA Mississippi State, MS 39762 U.S.A \\ dobson@math.msstate.edu \\ and \\ IAM \\ University of Primorska \\ 6000 Koper, Slovenia
}

Submitted: Feb 11, 2013; Accepted: Jul 4, 2014; Published: Jul 21, 2014

Mathematics Subject Classifications: 05E18, 05C25, 20F18

\begin{abstract}
We give a necessary condition to reduce the Cayley isomorphism problem for Cayley objects of a nilpotent or abelian group $G$ whose order satisfies certain arithmetic properties to the Cayley isomorphism problem of Cayley objects of the Sylow subgroups of $G$ in the case of nilpotent groups, and in the case of abelian groups to certain natural subgroups. As an application of this result, we show that $\mathbb{Z}_{q} \times \mathbb{Z}_{p}^{2} \times \mathbb{Z}_{m}$ is a CI-group with respect to digraphs, where $q$ and $p$ are primes with $p^{2}<q$ and $m$ is a square-free integer satisfying certain arithmetic conditions (but there are no other restrictions on $q$ and $p$ ).
\end{abstract}

Keywords: Cayley object; Cayley graph; isomorphism; CI-group

\section{Introduction}

In 1967 Ádám [1] conjectured that any two circulant graphs of order $n$ are isomorphic if and only if they are isomorphic by a group automorphism of $\mathbb{Z}_{n}$. While Ádám's conjecture was quickly shown to be false [4], the conjecture nonetheless generated much interest in the following question: Are two Cayley graphs of a group $G$ isomorphic if and only if they are isomorphic by a group automorphism of $G$ ? If so, we say that $G$ is a CIgroup with respect to graphs. This problem naturally generalizes to any class of 
combinatorial objects (see [11] for several equivalent formulations of the precise definition of a combinatorial object). Namely, is it true that two Cayley objects of a group $G$ in some class $\mathcal{K}$ of combinatorial objects are isomorphic if and only if they are isomorphic by a group automorphism of $G$ ? If so, we say that $G$ is a CI-group with respect to $\mathcal{K}$. If $G$ is a CI-group with respect to every class of combinatorial objects, we say that $G$ is a CI-group. In 1987, Pálfy [13] proved the following remarkable result:

Theorem 1. A group $G$ is a CI-group if and only if $\operatorname{gcd}(n, \varphi(n))=1$ or $n=4$, where $\varphi$ is Euler's phi function.

While Pálfy's result is quite powerful, it does not tell us anything in general about isomorphisms between Cayley objects of a group $G$ if $G$ is not a CI-group, other than there exists isomorphic Cayley objects of $G$ which are not isomorphic by a group automorphism of $G$. For such groups, we are then left with the question of if two Cayley objects of $G$ are isomorphic, then what are the possible isomorphisms between them? This is sometimes known as the Cayley isomorphism problem. Usually, one would like the solution to this question to be a (hopefully) short list $L$ of possible isomorphisms. That is, two Cayley objects of $G$ are isomorphic if and only if they are isomorphic by a function in the list $L$. In 1999, Muzychuk [11] showed that if $G$ is a cyclic group of order $n$ and for any distinct primes $p$ and $q$ dividing $n$ we have that $q$ does not divide $p-1$, then any two Cayley objects of $G$ are isomorphic by an automorphism that can be found in a natural way from isomorphisms of Cayley objects of prime-power orders that divide $n$. Thus Muzychuk reduced the Cayley isomorphism problem for Cayley objects of cyclic groups of some orders to the Cayley isomorphism problem for Cayley objects of cyclic groups of prime-power orders. In 2003, the author [3], found a sufficient condition to extend Muzychuk's result to all abelian groups (with the same order conditions), and showed this sufficient condition was satisfied by some abelian groups. In this paper, we extend the author's earlier result to nilpotent groups, as well as to abelian groups with more general order conditions (Theorem 14). Finally, as an application we will extend the list of CI-groups with respect to digraphs by showing that $\mathbb{Z}_{q} \times \mathbb{Z}_{p}^{2} \times \mathbb{Z}_{m}$ is a CI-group with respect to digraphs, where $p$ and $q$ are distinct primes with $p^{2}<q$ and $m$ satisfies certain arithmetic conditions (Theorem 31).

Throughout this paper, $G$ is a finite group. For group theoretic terms not defined in this paper, see [2]. We begin with some definitions.

Definition 2. Let $G$ be a transitive group acting on $\Omega$. Let $X$ be the set of all complete block systems of $G$. Define a partial order on $X$ by $\mathcal{B} \preceq \mathcal{C}$ if and only if every block of $\mathcal{C}$ is a union of blocks of $\mathcal{B}$. We define $\left.\mathcal{B}\right|_{C}$ to be the complete block system of $\operatorname{Stab}_{G}(C)=$ $\{g \in G: g(C)=C\}$, the set-wise stabilizer of $\boldsymbol{C} \in \mathcal{C}$, consisting of all those blocks of $\mathcal{B}$ that are contained in $C, C \in \mathcal{C}$, and remark that $\left.\mathcal{B}\right|_{C}$ is a complete block system of $\operatorname{Stab}_{G}(C)$ in its action on $C$. By $\operatorname{fix}_{G}(\mathcal{B})$ we mean the subgroup of $G$ which fixes each block of $\mathcal{B}$ set-wise. That is, $\operatorname{fix}_{G}(\mathcal{B})=\{g \in G: g(B)=B$ for all $B \in \mathcal{B}\}$. We denote by $\operatorname{Stab}_{G}(x)$ the stabilizer of $x \in X$. That is, $\operatorname{Stab}_{G}(x)=\{g \in G: g(x)=x\}$. Finally, $g \in G$ induces a natural permutation $g / \mathcal{B}$ in $S_{\mathcal{B}}$, and we set $G / \mathcal{B}=\{g / \mathcal{B}: g \in G\}$. 
Definition 3. Let $n=\prod_{i=1}^{r} p_{i}^{a_{i}}$ be the prime factorization of $n$ and define $\Omega: \mathbb{N} \mapsto \mathbb{N}$ by $\Omega(n)=\sum_{i=1}^{r} a_{i}$. Setting $m=\Omega(n)$, we say a transitive group $G$ of degree $n$ is $\boldsymbol{m}$-step imprimitive if there exists a sequence of complete block system $\mathcal{B}_{0} \prec \mathcal{B}_{1} \prec \ldots \prec \mathcal{B}_{m}$. Note that $\mathcal{B}_{0}$ consists of singletons, while $\mathcal{B}_{m}$ consists of the entire set on which $G$ acts. A complete block system $\mathcal{B}$ will be said to be normal if $\mathcal{B}$ is formed by the orbits of a normal subgroup. We will say that $G$ is normally $\boldsymbol{m}$-step imprimitive if each $\mathcal{B}_{i}$, $0 \leqslant i \leqslant m$, is formed by the orbits of a normal subgroup of $G$.

\section{The main tool}

In this section, we will give a sufficient condition that will imply that the Cayley isomorphism problem for nilpotent groups of certain orders can be reduced to the Cayley isomorphism problem for groups of prime-power order (Theorem 14 and Corollary 15), and for abelian groups with more general arithmetic conditions (Theorem 14). That these results have implications for the Cayley isomorphism problem is established in Theorem 26. The following result is straightforward, and so its proof is omitted.

Lemma 4. Let $G_{1}, G_{2} \leqslant S_{n}$ be transitive such that both $G_{1}$ and $G_{2}$ admit $\mathcal{B}$ as a complete block system. Then $\left\langle G_{1}, G_{2}\right\rangle$ admits $\mathcal{B}$ as a complete block system.

The following result is trivial after observing that the hypothesis implies that $\operatorname{fix}_{G}(\mathcal{B})=$ $\operatorname{Stab}_{G}(B)$.

Lemma 5. Let $G \leqslant S_{n}$ be m-step imprimitive with sequence $\mathcal{B}_{0}, \ldots, \mathcal{B}_{m}$. If $G / \mathcal{B}_{m-1}$ is cyclic of prime order $p$, then $\left.\operatorname{fix}_{G}\left(\mathcal{B}_{m-1}\right)\right|_{B}$ is $(m-1)$-step imprimitive for every $B \in \mathcal{B}_{m-1}$, with $(m-1)$-step imprimitivity sequence $\left.\mathcal{B}_{0}\right|_{B}, \ldots,\left.\mathcal{B}_{m-1}\right|_{B}$.

We will use the following basic (and known) result implicitly throughout the paper.

Lemma 6. Let $G \leqslant S_{n}$ be transitive with $H \leqslant G$ a transitive abelian subgroup. Then every complete block system of $G$ is normal and is formed by the orbits of a normal subgroup of $H$.

Proof. Let $\mathcal{B}$ be a complete block system of $G$ consisting of $m$ blocks of size $k$. As a transitive abelian group is regular [14, Proposition 1.4.4], we have that $H / \mathcal{B}$ is regular of degree $m$, so that $\operatorname{fix}_{H}(\mathcal{B}) \neq 1$ and has order $k$. As $\operatorname{Stab}_{H}(B)=\operatorname{fix}_{H}(\mathcal{B})$ for every $B \in \mathcal{B}$ and $\left.\operatorname{Stab}_{H}(B)\right|_{B}$ is transitive [2, Exercise 1.5.6], we have that $\left.\operatorname{fix}_{H}(\mathcal{B})\right|_{B}$ is transitive for every $B \in \mathcal{B}$. As the blocks of $\mathcal{B}$ have size $k$, we conclude that the orbits of $\operatorname{fix}_{H}(\mathcal{B}) \leqslant$ $\operatorname{fix}_{G}(\mathcal{B})$ form $\mathcal{B}$.

Definition 7. Let $G$ be a permutation group acting on $X$ and $H$ a permutation group acting on $Y$. Define the wreath product of $\boldsymbol{G}$ and $\boldsymbol{H}$, denoted $G \imath H$, to be the group of all permutations of $G \times H$ of the form $(x, y) \rightarrow\left(g(x), h_{x}(y)\right)$. 
Lemma 8. Let $n$ be a positive integer and $G_{1}, G_{2}$ be transitive abelian groups of degree $n$ such that $\left\langle G_{1}, G_{2}\right\rangle$ is m-step imprimitive. Let $n=p_{1}^{a_{1}} p_{2}^{a_{2}} \cdots p_{r}^{a_{r}}$ be the prime-power decomposition of $n$. Then there exists $\delta \in\left\langle G_{1}, G_{2}\right\rangle$ and a sequence of primes $q_{1}, \ldots, q_{m}$ such that $n=q_{1} \cdots q_{m}$ and $\left\langle G_{1}, \delta^{-1} G_{2} \delta\right\rangle$ is permutation isomorphic to a subgroup of $\operatorname{AGL}\left(1, q_{1}\right)$ ᄀ $\left.\left(\operatorname{AGL}\left(1, q_{2}\right) \curlywedge(\cdots) \operatorname{AGL}\left(1, q_{m}\right)\right)\right)$. Furthermore, if $\left\langle G_{1}, G_{2}\right\rangle$ is solvable, then we may take $\delta=1$.

Proof. We proceed by induction on $m$. If $m=1$, then $n$ is prime, and both $G_{1}$ and $G_{2}$ are Sylow $n$-subgroups of $S_{n}$. Hence there exists $\delta \in\left\langle G_{1}, G_{2}\right\rangle$ such that $\delta^{-1} G_{2} \delta=G_{1}$, and the result is trivial as $\left\langle G_{1}, \delta^{-1} G_{2} \delta\right\rangle$ is cyclic of order $n$. Now assume that the result is true for all $m-1 \geqslant 1$, and let $G_{1}, G_{2}$ be transitive abelian groups of degree $n$, where $\Omega(n)=m$, such that $\left\langle G_{1}, G_{2}\right\rangle$ is $m$-step imprimitive.

As $\left\langle G_{1}, G_{2}\right\rangle$ is $m$-step imprimitive, $\left\langle G_{1}, G_{2}\right\rangle$ admits a normal complete block system $\mathcal{B}$ consisting of $n / q_{m}$ blocks of size $q_{m}$ for some prime $q_{m} \mid n$, and both $G_{1} / \mathcal{B}$ and $G_{2} / \mathcal{B}$ are transitive abelian groups of degree $n / q_{m}$ and $\Omega\left(n / q_{m}\right)=m-1$. Furthermore, as $\left\langle G_{1}, G_{2}\right\rangle$ is $m$-step imprimitive, $\left\langle G_{1}, G_{2}\right\rangle / \mathcal{B}$ is $(m-1)$-step imprimitive by [3, Lemma 8], so by the induction hypothesis, there exists $\delta_{1} \in\left\langle G_{1}, G_{2}\right\rangle$ such that $\left\langle G_{1}, \delta_{1}^{-1} G_{2} \delta\right\rangle / \mathcal{B}$ is permutation isomorphic to a subgroup of $\operatorname{AGL}\left(1, q_{1}\right)$ ? $\left(\operatorname{AGL}\left(1, q_{2}\right)\right.$ ? $\left.\left.(\cdots) \operatorname{AGL}\left(1, q_{m-1}\right)\right)\right)$ for some sequence of primes $q_{1}, \ldots, q_{m-1}$ such that $n / q_{m}=q_{1} \cdots q_{m-1}$, and if $\left\langle G_{1}, G_{2}\right\rangle$ is solvable, we may take $\delta_{1}=1$. Furthermore, fix $\operatorname{lix}_{G_{1}}(\mathcal{B})$ is semiregular of order $q_{m}$, and $\operatorname{fix}_{\delta_{1}^{-1} G_{2} \delta_{1}}(\mathcal{B})$ is also semiregular of order $q_{m}$. Hence there exists $\delta_{2} \in \operatorname{fix}_{\left\langle G_{1}, \delta_{1}^{-1} G_{2} \delta_{1}\right\rangle}(\mathcal{B})$ such that $\delta_{2}^{-1} \operatorname{fix}_{\delta_{1}^{-1} G_{2} \delta_{1}}(\mathcal{B}) \delta_{2}$ is contained in the same Sylow $q_{m}$-subgroup of $\operatorname{fix}_{\left\langle G_{1}, \delta_{1}^{-1} G_{2} \delta_{1}\right\rangle}(\mathcal{B})$ as $\operatorname{fix}_{G_{1}}(\mathcal{B})$. If $\left\langle G_{1}, G_{2}\right\rangle$ is solvable, then $\operatorname{fix}_{\left\langle G_{1}, G_{2}\right\rangle}(\mathcal{B})$ is solvable, so $\left.\operatorname{fix}_{\left\langle G_{1}, G_{2}\right\rangle}(\mathcal{B})\right|_{B}$ is solvable, and by [2, Exercise 3.5.1], fix $\left.\left\langle G_{1}, G_{2}\right\rangle(\mathcal{B})\right|_{B}$ has a unique Sylow $q_{m}$-subgroup, so we may take $\delta_{2}=1$. Let $\delta=\delta_{1} \delta_{2}$. As a Sylow $q_{m}$-subgroup of $\operatorname{fix}_{\left\langle G, \delta^{-1} G \delta\right\rangle}(\mathcal{B})$ is contained in $1_{S_{n / q_{m}}} \prec \mathbb{Z}_{q_{m}}$ we have that both $G_{1}$ and $\delta^{-1} G_{2} \delta$ normalize $1_{S_{n / q_{m}}} \prec \mathbb{Z}_{q_{m}}$. This then implies that $\left.\operatorname{Stab}_{\left\langle G_{1}, \delta^{-1} G_{2} \delta\right\rangle}(B)\right|_{B}$ has a normal Sylow $q_{m}$-subgroup, so that $\left.\operatorname{Stab}_{\left\langle G_{1}, \delta^{-1} G_{2} \delta\right\rangle}(B)\right|_{B}$ is permutation isomorphic to a subgroup of $\operatorname{AGL}\left(1, q_{m}\right)$ for every $B \in \mathcal{B}$. It then follows by the Embedding Theorem [9, Theorem 2.6], that $\left\langle G_{1}, \delta^{-1} G_{2} \delta\right\rangle$ is permutation isomorphic to a subgroup of $\operatorname{AGL}\left(1, q_{1}\right)$ ? $\left.\left(\operatorname{AGL}\left(1, q_{2}\right) \imath(\cdots) \operatorname{AGL}\left(1, q_{m}\right)\right)\right)$, and the result follows by induction.

Definition 9. Let $\pi$ be a set of primes. A $\pi$-group $G$ is a group such that every prime divisor of $|G|$ is contained in $\pi$. A subgroup $H$ of $G$ is an $\boldsymbol{S}_{\boldsymbol{\pi}}$-subgroup of $\boldsymbol{G}$ if no prime in $\pi$ divides $|G| /|H|$. By $\pi^{\prime}$, we denote the set of primes dividing $|G|$ that are not contained in $\pi$.

We shall have need a consequence of the preceding result.

Lemma 10. Let $n$ be a positive integer and $\pi$ be the set of distinct prime numbers dividing $n$. If $G_{1}, G_{2}$ are transitive abelian groups of degree $n$ such that $\left\langle G_{1}, G_{2}\right\rangle$ is m-step imprimitive then there exists $\delta \in\left\langle G_{1}, G_{2}\right\rangle$ such that $\left\langle G_{1}, \delta^{-1} G_{2} \delta\right\rangle$ is a solvable $\pi$-group.

Proof. Let $n=p_{1}^{a_{1}} \cdots p_{r}^{a_{r}}$ be the prime-power decomposition of $n$. By Lemma 8, there exists $\delta_{1} \in\left\langle G_{1}, G_{2}\right\rangle$ and a sequence $q_{1}, \ldots, q_{m}$ of primes such that $n=q_{1} \cdots q_{m}$ and 
$\left\langle G_{1}, \delta_{1}^{-1} G_{2} \delta_{1}\right\rangle \leqslant \operatorname{AGL}\left(1, q_{1}\right) \succ\left(\operatorname{AGL}\left(1, q_{2}\right) \imath\left(\cdots>\operatorname{AGL}\left(1, q_{m}\right)\right)\right)$. As $\operatorname{AGL}\left(1, q_{i}\right)$ is solvable for every $1 \leqslant i \leqslant m,\left\langle G_{1}, \delta_{1}^{-1} G_{2} \delta_{1}\right\rangle$ is solvable. By Hall's Theorem [5, Theorem 6.4.1], we have that $G_{1}$ is contained in an $S_{\pi}$-subgroup $H_{1}$ of $\left\langle G_{1}, \delta_{1}^{-1} G_{2} \delta_{1}\right\rangle$ and that $G_{2}$ is

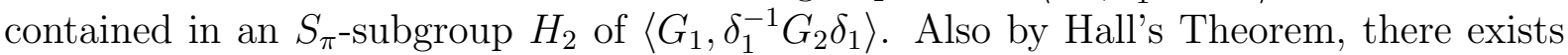
$\delta_{2} \in\left\langle G_{1}, \delta_{1}^{-1} G_{2} \delta_{1}\right\rangle$ such that $\delta_{2}^{-1} H_{2} \delta=H_{1}$. Let $\delta=\delta_{1} \delta_{2}$. Then $\left\langle G_{1}, \delta^{-1} G_{2} \delta\right\rangle \leqslant H_{1}$, and $H_{1}$ is a solvable $\pi$-group.

Let $\mathcal{L}=\mathcal{L}_{G}$ be the set of all normal complete block systems of a transitive group $G$. Then $\preceq$ is a canonical partial order on $\mathcal{L}$. Define operations $\cup$ and $\cap$ on $\mathcal{L}$ by $\mathcal{B} \cup \mathcal{C}$ is the normal complete block system of $G$ formed by the orbits of $\left\langle\operatorname{fix}_{G}(\mathcal{B}), \operatorname{fix}_{G}(\mathcal{C})\right\rangle=$ $\operatorname{fix}_{G}(\mathcal{B}) \cdot \operatorname{fix}_{G}(\mathcal{C})$ (as both of these groups are normal), and $\mathcal{B} \cap \mathcal{C}$ is the normal complete block system of $G$ formed by the orbits of $\operatorname{fix}_{G}(\mathcal{B}) \cap \operatorname{fix}_{G}(\mathcal{C})$. Notice that both of these operation do in fact give normal complete block systems as $\left\langle\operatorname{fix}_{G}(\mathcal{B}), \operatorname{fix}_{G}(\mathcal{C})\right\rangle \triangleleft G$ and $\operatorname{fix}_{G}(\mathcal{G}) \cap \operatorname{fix}_{G}(\mathcal{C}) \triangleleft G$. Thus $\mathcal{L}_{G}$ is a lattice. See [6] for terms regarding lattices not defined here. We also have that

Lemma 11. If $G$ contains a transitive abelian group $H$, then $\mathcal{L}_{G}$ is a modular lattice.

Proof. We must show that if $\mathcal{B} \preceq \mathcal{A}$, then $\mathcal{A} \cap(\mathcal{B} \cup \mathcal{C})=\mathcal{B} \cup(\mathcal{A} \cap \mathcal{C})$. By Lemma 6, there exists $A, B, C \leqslant H$ such that $\mathcal{A}$ is formed by the orbits of $A, \mathcal{B}$ is formed by the orbits of $B$, and $\mathcal{C}$ is formed by the orbits of $C$. As $\mathcal{B} \preceq \mathcal{A}$, we have that $B \leqslant A$. By $[6$, Theorem 8.4.1], we have that $A \cap(B \cdot C)=B \cdot(A \cap C)$. Then the orbits of $A \cap(B \cdot C)$ are the same as the orbits of $B \cdot(A \cap C)$.

We remark that the previous result is contained in [12, Theorem 2.10]

In the following three results, we will have in the hypothesis that $\operatorname{gcd}\left(n_{i}, n_{j} \cdot \varphi\left(n_{j}\right)\right)=1$. Notice that this implies that $\operatorname{gcd}\left(n_{i}, n_{j}\right)=1$, and that if $p_{i} \mid n_{i}$ is prime, then $p_{i}$ does not divide $p_{j}-1$ for any prime $p_{j} \mid n_{j}$.

Lemma 12. Let $n_{1}, \ldots, n_{r}$ be positive integers such that if $i \neq j$, then $\operatorname{gcd}\left(n_{i}, n_{j} \cdot \varphi\left(n_{j}\right)\right)=$ $1, \pi_{i}$ the set of primes dividing $n_{i}$, and $H_{i}$ be a transitive, solvable, $\pi_{i}$-group of degree $n_{i}$, $1 \leqslant i \leqslant r$. Let $G$ be a transitive $m$-step imprimitive subgroup of $\prod_{i=1}^{r} H_{i}$ acting coordinatewise, where $\Omega\left(n_{1} \cdots n_{r}\right)=m$. Then there exists transitive subgroups $L_{i} \leqslant H_{i}$ such that $G=\prod_{i=1}^{r} L_{i}$.

Proof. It is not difficult to see that $\prod_{i=1}^{r} H_{i}$ admits complete block systems $\mathcal{C}_{i}$ consisting of $n / n_{i}$ blocks of size $n_{i}, 1 \leqslant i \leqslant r$, formed by the orbits of $H_{i}$. As each $H_{i}$ is solvable, we have that $G$ is solvable, and so contains an $S_{\pi_{i}}$-subgroup $L_{i}$ for every $1 \leqslant i \leqslant r$. As $\Pi_{i=1}^{r} H_{i} / \mathcal{C}_{i}$ is a $\pi_{i}^{\prime}$-group, $G / \mathcal{C}_{i}$ is also a $\pi_{i}^{\prime}$-group, and so fix $\mathcal{C}_{G}\left(\mathcal{C}_{i}\right)=L_{i}$. Then $L_{i} \cap L_{j}=1$ for every $i \neq j, L_{i} \triangleleft G$, and $\left\langle L_{i}: 1 \leqslant i \leqslant r\right\rangle=G$. The result now follows.

We now need only one more tool to prove the main result (Theorem 14) of this section. Before proceeding to this last tool, it will be useful to develop some terminology which will simplify the statement. First, the proof of Theorem 14 will proceed by induction on $m=\Omega(n)$. So when proving Theorem 14, we will be assuming that the conclusion of Theorem 14 holds for all integers $n / p$, where $p$ divides $n$ is prime. In particular, with 
$m, n_{1}, \ldots, n_{r}$ and $n$ satisfying the hypothesis of Theorem 14 and $G_{1}, G_{2}$ transitive abelian or nilpotent groups of degree $n$, then whenever $K_{1}, K_{2}$ are transitive nilpotent or abelian groups of degree $n / p$ - and to simplify our notation, there is no harm in assuming that $p \mid n_{1}$ - such that $\left\langle K_{1}, K_{2}\right\rangle$ are $(m-1)$-step imprimitive, then there exists $\delta \in\left\langle K_{1}, K_{2}\right\rangle$ such that $\left\langle K_{1}, \delta^{-1} K_{2} \delta\right\rangle \leqslant \Pi_{i=1}^{r} \bar{H}_{i}$, where $\bar{H}_{i}$ is a solvable $\bar{\pi}_{i}$ group of degree $\bar{n}_{i}$. Here $\bar{n}_{i}=n_{i}$ if $i \neq 1$ while $\bar{n}_{1}=n_{1} / p$, and $\bar{\pi}_{i}$ is the set of prime divisors of $\bar{n}_{i}$. In this situation, we will say that $n$ satisfies the main induction hypothesis.

Lemma 13. Let $n_{1}, \ldots, n_{r}$ be positive integers such that if $i \neq j$, then $\operatorname{gcd}\left(n_{i}, n_{j} \cdot \varphi\left(n_{j}\right)\right)=$ 1. Let $n=n_{1} \cdots n_{r}$ with $\Omega(n)=m, p \mid n$ (and without loss of generality, $p \mid n_{1}$ ), and $\bar{\pi}=\cup_{j \in I} \bar{\pi}_{j}$ for some $I \subseteq[r]$. If

1. $n$ satisfies the main induction hypothesis,

2. $\left\langle G_{1}, G_{2}\right\rangle$ is m-step imprimitive,

3. $\left\langle G_{1}, G_{2}\right\rangle$ admits a complete block system $\mathcal{B}$ of $p$ blocks of size $n / p$, and

4. $\left\langle G_{1}, G_{2}\right\rangle / \mathcal{B}$ is a p-group,

then there exists $\delta \in\left\langle G_{1}, G_{2}\right\rangle$ such that $\left\langle G_{1}, \delta^{-1} G_{2} \delta\right\rangle$ admits a complete block system formed by the orbits of the unique $S_{\bar{\pi}}$-subgroup of $\operatorname{fix}_{G_{i}}(\mathcal{B}), i=1,2$.

Proof. Let $B \in \mathcal{B}$ and $K_{i}=\left.\operatorname{fix}_{G_{i}}(\mathcal{B})\right|_{B}, i=1,2$. As $\left\langle G_{1}, G_{2}\right\rangle / \mathcal{B}$ has prime order $p$, we must have that $\operatorname{Stab}_{\left\langle G_{1}, G_{2}\right\rangle}(B)=\operatorname{fix}_{\left\langle G_{1}, G_{2}\right\rangle}(\mathcal{B})$. Note that $K_{i}$ is transitive on $B$, and if $G_{i}$ is nilpotent or abelian, then $K_{i}$ is nilpotent or abelian, $i=1,2$. Clearly $\operatorname{fix}_{G_{1}}(\mathcal{B})$, $\operatorname{fix}_{G_{2}}(\mathcal{B}) \leqslant$ $\operatorname{fix}_{\left\langle G_{1}, G_{2}\right\rangle}(\mathcal{B})$. By Lemma $5,\left.\operatorname{fix}_{\left\langle G_{1}, G_{2}\right\rangle}(\mathcal{B})\right|_{B}$ is $(m-1)$-step imprimitive, so that $\left\langle K_{1}, K_{2}\right\rangle$ is $(m-1)$-step imprimitive.

By hypothesis, there exists $\delta_{1} \in\left\langle\operatorname{fix}_{G_{1}}(\mathcal{B}), \operatorname{fix}_{G_{2}}(\mathcal{B})\right\rangle$ such that

$$
\left.\left\langle\operatorname{fix}_{G_{1}}(\mathcal{B}), \delta_{1}^{-1} \operatorname{fix}_{G_{2}}(\mathcal{B}) \delta_{1}\right\rangle\right|_{B} \leqslant \Pi_{j=1}^{r} \bar{H}_{B, j},
$$

where each $\bar{H}_{B, j}$ is a transitive solvable $\bar{\pi}_{j}$-group of degree $\bar{n}_{j}$. Similarly, if $B \neq B^{\prime} \in \mathcal{B}$, then there exists $\delta_{2} \in\left\langle\operatorname{fix}_{G_{1}}(\mathcal{B}), \delta_{1}^{-1} \operatorname{fix}_{G_{2}}(\mathcal{B}) \delta_{1}\right\rangle$ such that

$$
\left.\left\langle\operatorname{fix}_{G_{1}}(\mathcal{B}), \delta_{2}^{-1} \delta_{1}^{-1} \operatorname{fix}_{G_{2}}(\mathcal{B}) \delta_{1} \delta_{2}\right\rangle\right|_{B^{\prime}} \leqslant \Pi_{j=1}^{r} \bar{H}_{B^{\prime}, j},
$$

where each $\bar{H}_{B^{\prime}, j}$ is a transitive solvable $\bar{\pi}_{j}$-group of degree $\bar{n}_{j}$. Furthermore, we have that $\left.\left.\delta_{2}\right|_{B} \in\left\langle\operatorname{fix}_{G_{1}}(\mathcal{B}), \delta_{1}^{-1} \operatorname{fix}_{G_{2}}(\mathcal{B}) \delta_{1}\right\rangle\right|_{B}$. This then implies that

$$
\left.\left\langle\operatorname{fix}_{G_{1}}(\mathcal{B}), \delta_{2}^{-1} \delta_{1}^{-1} \operatorname{fix}_{G_{2}}(\mathcal{B}) \delta_{1} \delta_{2}\right\rangle\right|_{B} \leqslant\left.\left\langle\operatorname{fix}_{G_{1}}(\mathcal{B}), \delta_{1}^{-1} \operatorname{fix}_{G_{2}}(\mathcal{B}) \delta_{1}\right\rangle\right|_{B} .
$$

Hence $\left.\left\langle\operatorname{fix}_{G_{1}}(\mathcal{B}), \delta_{2}^{-1} \delta_{1}^{-1} \operatorname{fix}_{G_{2}}(\mathcal{B}) \delta_{1} \delta_{2}\right\rangle\right|_{B} \leqslant \Pi_{j=1}^{r} \bar{H}_{B, j}$. Continuing inductively, we have that there exists $\delta \in\left\langle\operatorname{fix}_{G_{1}}(\mathcal{B})\right.$, $\left.\operatorname{fix}_{G_{2}}(\mathcal{B})\right\rangle$ such that $\left.\left\langle\operatorname{fix}_{G_{1}}(\mathcal{B}), \delta^{-1} \operatorname{fix}_{G_{2}}(\mathcal{B}) \delta\right\rangle\right|_{B} \leqslant \Pi_{j=1}^{r} \bar{H}_{B, j}$ for every $B \in \mathcal{B}$, where each $\bar{H}_{B, j}$ is a transitive solvable $\bar{\pi}_{j}$-group of degree $\bar{n}_{j}$. Note that $\delta^{-1} \operatorname{fix}_{G_{2}}(\mathcal{B}) \delta=\operatorname{fix}_{\delta^{-1} G_{2} \delta}(\mathcal{B})$ as $\delta / \mathcal{B}=1$. For ease of notation, we will replace $\delta^{-1} G_{2} \delta$ by $G_{2}$ and thus assume without loss of generality that $\left.\left\langle\operatorname{fix}_{G_{1}}(\mathcal{B}), \operatorname{fix}_{G_{2}}(\mathcal{B})\right\rangle\right|_{B} \leqslant$ 
$\Pi_{j=1}^{r} H_{B, j}$ for each $B \in \mathcal{B}$. By Lemma 12 , we may assume without loss of generality that $\left.\left\langle\operatorname{fix}_{G_{1}}(\mathcal{B}), \operatorname{fix}_{G_{2}}(\mathcal{B})\right\rangle\right|_{B}=\prod_{j=1}^{r} \bar{H}_{B, j}$ for each $B \in \mathcal{B}$.

As $\left.\left\langle\operatorname{fix}_{G_{1}}(\mathcal{B}), \operatorname{fix}_{G_{2}}(\mathcal{B})\right\rangle\right|_{B}=\prod_{j=1}^{r} H_{B, j}$ for each $B \in \mathcal{B},\left.\left\langle\operatorname{fix}_{G_{1}}(\mathcal{B}), \operatorname{fix}_{G_{2}}(\mathcal{B})\right\rangle\right|_{B}$ has a normal subgroup $L_{B}$ with orbits of size $\prod_{j \in I} \bar{n}_{j}$, and so $\left.\left\langle\operatorname{fix}_{G_{1}}(\mathcal{B})\right.$, fix $\left.G_{G_{2}}(\mathcal{B})\right\rangle\right|_{B}$ admits a complete block system $\mathcal{C}_{B}$ formed by the orbits of $L_{B}, B \in \mathcal{B}$. Also note that the $S_{\bar{\pi}^{-}}$ subgroup of $\left.\operatorname{fix}_{G_{i}}(\mathcal{B})\right|_{B}$ must be contained in $L_{B}$, as otherwise $\left(\left.\operatorname{fix}_{G_{i}}(\mathcal{B})\right|_{B}\right) / \mathcal{C}_{B}$ contains a nontrivial normal subgroup with orbits of size dividing $\prod_{j \in I} \bar{n}_{j}, i=1,2$. However, $\left(\left.\operatorname{fix}_{G_{i}}(\mathcal{B})\right|_{B}\right) / \mathcal{C}_{B}$ is a solvable $\bar{\pi}^{\prime}$-subgroup and $\operatorname{gcd}\left(\Pi_{j \in I} \bar{n}_{j}, \Pi_{j \notin I, j \in[r]} \bar{n}_{j}\right)=1, i=1,2$, a contradiction. Then $\left.\operatorname{fix}_{G_{i}}(\mathcal{B})\right|_{B}, i=1,2$, admit complete block systems $\mathcal{D}_{B}$ formed by the orbits of their unique $S_{\bar{\pi}}$-subgroups, respectively, and these complete block systems must be precisely $\mathcal{C}_{B}$, for $B \in \mathcal{B}$.

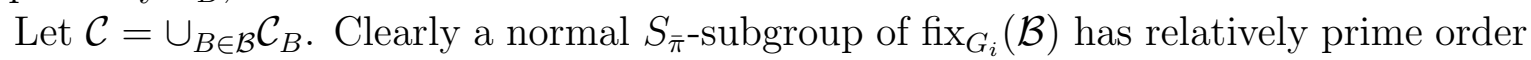

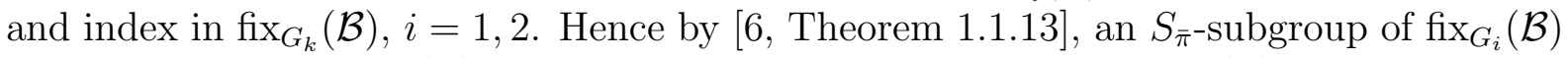

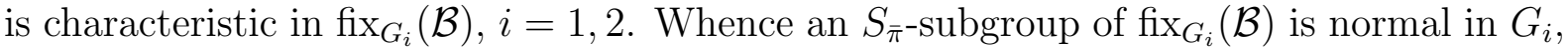
$i=1,2$. Thus $G_{i}$ admits complete block systems formed by the orbits of the $S_{\bar{\pi}}$-subgroup of $\operatorname{fix}_{G_{i}}(\mathcal{B}), i=1,2$. As each $\mathcal{C}_{B}$ is formed by the orbits of the $S_{\bar{\pi}}$-subgroup of fix $_{G_{i}}(\mathcal{B})$

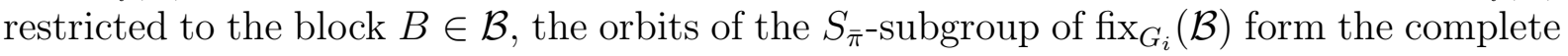
block system $\mathcal{C}, i=1,2$. Hence $\mathcal{C}$ is a block system of $G_{i}, i=1,2$, and so by Lemma 4 , $\mathcal{C}$ is a complete block system of $\left\langle G_{1}, G_{2}\right\rangle$.

We now prove the main result of this section.

Theorem 14. Let $n_{1}, \ldots, n_{r}$ be positive integers such that $\operatorname{gcd}\left(n_{i}, n_{j} \cdot \varphi\left(n_{j}\right)\right)=1$ if $i \neq j$, and $\pi_{i}$ be the set of distinct prime numbers dividing $n_{i}$. Let $n=n_{1} \cdots n_{r}$ and $m=\Omega(n)$. If either

1. each $n_{i}$ is a prime-power, and $G_{1}, G_{2}$ are transitive nilpotent groups of degree $n$ such that $\left\langle G_{1}, G_{2}\right\rangle$ is m-step imprimitive, or

2. $G_{1}, G_{2}$ are transitive abelian groups of degree $n$ such that $\left\langle G_{1}, G_{2}\right\rangle$ is m-step imprimitive,

then there exists $\delta \in\left\langle G_{1}, G_{2}\right\rangle$ such that $\left\langle G_{1}, \delta^{-1} G_{2} \delta\right\rangle=\Pi_{i=1}^{r} H_{i}$, where each $H_{i}$ is a transitive solvable $\pi_{i}$-group of degree $n_{i}$.

Proof. Throughout the proof, if case (1) holds, we let $p_{i}$ be prime such that $n_{i}=p_{i}^{a_{i}}$, $a_{i} \geqslant 1$. First suppose that case (1) holds and $r=1$. Then $G_{1} \leqslant \Pi_{1}, G_{2} \leqslant \Pi_{2}$, where $\Pi_{1}, \Pi_{2}$ are Sylow $p_{1}$-subgroups of $\left\langle G_{1}, G_{2}\right\rangle$. By a Sylow Theorem, there exists $\delta \in\left\langle G_{1}, G_{2}\right\rangle$ such that $\delta^{-1} G_{2} \delta \leqslant \Pi_{1}$. Then $\left\langle G_{1}, \delta^{-1} G_{2} \delta\right\rangle$ is a $p_{1}$-group and so nilpotent.

In both cases, we proceed by induction on $m$. Suppose that $m=1$. Then the only case that occurs is case (1) and $r=1$. The result then follows by arguments above. Assume that the result is true for all $G_{1}$ and $G_{2}$ that satisfy the hypothesis with $\Omega(n)=m-1 \geqslant 1$, and let $G_{1}, G_{2} \leqslant S_{n}$ satisfy the hypothesis where $\Omega(n)=m$. In case (1), by arguments above, we may assume that $r \geqslant 2$. In case (2), if $r=1$ then the result follows from Lemma 10 , so in any case we may assume without loss of generality that $r \geqslant 2$. Let $\mathcal{B}_{1}$ 
be a complete block system of $\left\langle G_{1}, G_{2}\right\rangle$ consisting of $p_{i}$ blocks of size $n / p_{i}$, where $p_{i} \mid m_{i}$. Then $\mathcal{B}_{1}$ is a complete block system of both $G_{1}$ and $G_{2}$. As both $G_{1}$ and $G_{2}$ are nilpotent, $G_{1} / \mathcal{B}_{1}$ and $G_{2} / \mathcal{B}_{1}$ are nilpotent. We conclude that both $G_{1} / \mathcal{B}_{1}$ and $G_{2} / \mathcal{B}_{1}$ are $p_{i}$-groups. Hence there exists $\delta_{1} \in\left\langle G_{1}, G_{2}\right\rangle$ such that $\left\langle G_{1}, \delta^{-1} G_{1} \delta\right\rangle / \mathcal{B}_{1}$ has order $p_{i}$. We thus assume without loss of generality that $\left\langle G_{1}, G_{2}\right\rangle / \mathcal{B}_{1}$ has order $p_{i}$.

Let $\pi=\cup_{j \in J} \pi_{j}$ where $J=[r]-\{i\}$. As $\left|G_{1} / \mathcal{B}_{1}\right|=\left|G_{2} / \mathcal{B}_{1}\right|=p_{i}$, the $S_{\pi^{-}}$subgroups of $G_{1}$ and $G_{2}$ are contained in $\left.\left.\operatorname{fix}_{\left\langle G_{1}, G_{2}\right\rangle}\right\rangle \mathcal{B}_{1}\right)$. By Lemma 13 and the induction hypothesis, there exists $\delta_{2} \in\left\langle G_{1}, G_{2}\right\rangle$ such that $\left\langle G_{1}, \delta_{2}^{-1} G_{2} \delta_{2}\right\rangle$ admits a complete block system $\mathcal{C}$ of $n_{i}$ blocks of size $n / n_{i}$ formed by the $S_{\pi}$-subgroups of $G_{1}$ and $G_{2}$. We thus assume without loss of generality that $\left\langle G_{1}, G_{2}\right\rangle$ admits $\mathcal{C}$ as a complete block system. Similarly, by Lemma 13 and the induction hypothesis, there exists $\delta_{3} \in\left\langle G_{1}, G_{2}\right\rangle$ such that $\left\langle G_{1}, \delta_{3}^{-1} G_{2} \delta_{3}\right\rangle$ admits a complete block system $\mathcal{D}$ formed by the orbits of an $S_{\pi_{i}}$-subgroup of fix f $_{G_{k}}\left(\mathcal{B}_{1}\right), k=1,2$, (we remark that if $n_{i}$ is prime, then $\mathcal{D}$ is trivial). We thus also assume without loss of generality that $\left\langle G_{1}, G_{2}\right\rangle$ admits $\mathcal{D}$ as well.

If $n_{i} \neq p_{i}$, then $\left\langle G_{1}, G_{2}\right\rangle / \mathcal{D}$ is $\left(m-\left(a_{i}-1\right)\right)$-step imprimitive, where $\Omega\left(n_{i}\right)=a_{i}$, and $G_{k} / \mathcal{D}$ is nilpotent, $k=1,2$. Hence by the induction hypothesis there exists $\delta_{4} \in\left\langle G_{1}, G_{2}\right\rangle$ such that $\left\langle G_{1}, \delta_{4}^{-1} G_{2} \delta_{4}\right\rangle / \mathcal{D} \leqslant P_{i} \times \prod_{j=1, j \neq i}^{r} H_{j}^{\prime}$, where $H_{j}^{\prime} \leqslant S_{n_{j}}$ is a transitive solvable $\pi_{j}$-group, and $P_{i}$ is a $p_{i}$-group of degree $p_{i}$. Then $\left\langle G_{1}, \delta_{4}^{-1} G_{2} \delta_{4}\right\rangle / \mathcal{D}$ admits a complete block system $\mathcal{E}^{\prime}$ of $n /\left(n_{i} / p_{i}\right)$ blocks of size $p_{i}$, so that $\left\langle G_{1}, \delta_{4}^{-1} G_{2} \delta_{4}\right\rangle$ admits a complete block system $\mathcal{E}$ of $n / n_{i}$ blocks of size $n_{i}$.

If $n_{i}=p_{i}$, then as $\left\langle G_{1}, G_{2}\right\rangle$ is $m$-step imprimitive, $\left\langle G_{1}, G_{2}\right\rangle$ admits a complete block system $\mathcal{B}_{2}$ such that $\mathcal{B}_{2} \preceq \mathcal{B}_{1}$ and $\mathcal{B}_{2}$ consists of $p_{i} p_{j}$ blocks of size $n /\left(p_{i} p_{j}\right)$ for some $p_{j} \mid n_{j}$ with $j \neq i$. Then $G_{1} / \mathcal{B}_{2}$ and $G_{2} / \mathcal{B}_{2}$ are nilpotent and transitive. We conclude that $G_{1} / \mathcal{B}_{2}$ and $G_{2} / \mathcal{B}_{2}$ are cyclic. By Theorem 1 , there exists $\delta_{3} \in\left\langle G_{1}, G_{2}\right\rangle$ such that $\left\langle G_{1}, \delta_{3}^{-1} G_{2} \delta_{3}\right\rangle / \mathcal{B}_{2}$ is cyclic. We thus assume without loss of generality that $\left\langle G_{1}, G_{2}\right\rangle / \mathcal{B}_{2}$ is cyclic. Thus $\left\langle G_{1}, G_{2}\right\rangle / \mathcal{B}_{2}$ admits a complete block system of $p_{j}$ blocks of size $p_{i}$, so that $\left\langle G_{1}, G_{2}\right\rangle$ admits a complete block system $\mathcal{B}_{1}^{\prime}$ of $p_{j}$ blocks of size $n / p_{j}$, and by Lemma 5 $\left.\operatorname{fix}_{\left\langle G_{1}, G_{2}\right\rangle}\left(\mathcal{B}_{1}^{\prime}\right)\right|_{B^{\prime}}$ is $(m-1)$-step imprimitive for every $B^{\prime} \in \mathcal{B}_{1}^{\prime}$. Hence by Lemma 13 , there exists $\delta_{4} \in\left\langle G_{1}, G_{2}\right\rangle$ such that $\left\langle G_{1}, \delta_{4}^{-1} G_{2} \delta_{4}\right\rangle$ admits a complete block system $\mathcal{E}$ of $n / n_{i}$ blocks of size $n_{i}$ formed by the orbits of an $S_{\pi_{i}}$-subgroup of fix $\operatorname{Gi}_{G_{k}}\left(\mathcal{B}_{1}^{\prime}\right), k=1,2$. Hence regardless of the value of $n_{i}$, we may assume without loss of generality that $\left\langle G_{1}, G_{2}\right\rangle$ admits $\mathcal{C}$ and $\mathcal{E}$ as complete block systems. As $\left\langle G_{1}, G_{2}\right\rangle$ admits a complete block system $\mathcal{C}$ of $n_{i}$ blocks of size $n / n_{i},\left\langle G_{1}, G_{2}\right\rangle \leqslant S_{n_{i}} 2 S_{n / n_{i}}$. As $\left\langle G_{1}, G_{2}\right\rangle$ also admits $\mathcal{E}$ as a complete block system and $\operatorname{gcd}\left(n_{i}, n / n_{i}\right)=1$, we have that $\left\langle G_{1}, G_{2}\right\rangle \leqslant S_{n / n_{i}} 2 S_{n_{i}}$. We conclude that $\left\langle G_{1}, G_{2}\right\rangle \leqslant\left(S_{n_{i}} \succ S_{n / n_{i}}\right) \cap\left(S_{n / n_{i}} 2 S_{n_{i}}\right)=S_{n_{i}} \times S_{n / n_{i}}$. We now consider (1) and (2) separately.

(1) By the induction hypothesis, we may, after a suitable conjugation, assume that $\left\langle G_{1}, G_{2}\right\rangle / \mathcal{D} \leqslant \Pi_{j \in[r]-\{i\}} S_{m_{j}}$, so that $\left\langle G_{1}, G_{2}\right\rangle \leqslant \Pi_{j=1}^{r} S_{m_{j}}$. The result then follows by inductively applying a Sylow Theorem and then Lemma 12.

(2) By Lemma 6 every complete block system is a normal complete block system. As $\mathcal{L}_{\left\langle G_{1}, G_{2}\right\rangle}$ is a modular lattice by Lemma 11, it follows by the Jordan-Dedekind Chain Condition [6, pg. 119] that all finite chains between two elements have the same length. As $\left\langle G_{1}, G_{2}\right\rangle$ admits $\mathcal{E}$ as a complete block system, any maximal chain between the complete 
block systems consisting of singletons and the complete block system consisting of one block that contain $\mathcal{E}$ must have length $m$ as $\left\langle G_{1}, G_{2}\right\rangle$ is normally $m$-step imprimitive. We conclude that $\left\langle G_{1}, G_{2}\right\rangle / \mathcal{E}$ is $\left(m-a_{i}\right)$-step imprimitive, so by the induction hypothesis we may assume after a suitable conjugation that $\left\langle G_{1}, G_{2}\right\rangle / \mathcal{E} \leqslant \Pi_{j=1, j \neq i}^{r} H_{j}$, where $H_{j} \leqslant S_{n_{j}}$ is a transitive solvable $\pi_{j}$-group. Similarly, we may assume that $\left\langle G_{1}, G_{2}\right\rangle / \mathcal{C} \leqslant H_{i}$, where $H_{i}$ is a transitive solvable $\pi_{i}$-group. As $C \cap E$ is a singleton, for every $C \in \mathcal{C}, E \in \mathcal{E}$, we have that $\left\langle G_{1}, G_{2}\right\rangle \leqslant \Pi_{j=1}^{r} H_{j}$. By Lemma 12 , we may assume that $\left\langle G_{1}, G_{2}\right\rangle=\prod_{j=1}^{r} H_{j}$ as

required. The result then follows by induction.

It may be worthwhile restating Theorem 14 (1) in the following form:

Corollary 15. Let $n=p_{1}^{a_{1}} \cdots p_{r}^{a_{r}}$, the prime-power decomposition of $n$, be such that $p_{i} \Lambda\left(p_{j}-1\right), 1 \leqslant i, j \leqslant r$. Let $\Omega(n)=m$. If $G_{1}, G_{2}$ are transitive nilpotent groups of degree $n$ such that $\left\langle G_{1}, G_{2}\right\rangle$ is m-step imprimitive, then there exists $\delta \in\left\langle G_{1}, G_{2}\right\rangle$ such that $\left\langle G_{1}, \delta^{-1} G_{2} \delta\right\rangle$ is nilpotent.

\section{Solving Sets}

In this section, we further develop the terminology regarding solving sets as well as the characterizations of when a particular set is a solving set that will be needed for our main results.

Definition 16. Let $G$ be a group and define $g_{L}: G \rightarrow G$ by $g_{L}(x)=g x$. Let $G_{L}=$ $\left\{g_{L}: g \in G\right\}$. Then $G_{L}$ is the left-regular representation of $\boldsymbol{G}$. We define a Cayley object of $\boldsymbol{G}$ to be a combinatorial object $X$ (e.g. digraph, graph, design, code) such that $G_{L} \leqslant \operatorname{Aut}(X)$, where $\operatorname{Aut}(X)$ is the automorphism group of $\boldsymbol{X}$ (note that this implies that the vertex set of $X$ is in fact $G$ ). If $X$ is a Cayley object of $G$ in some class $\mathcal{K}$ of combinatorial objects with the property that whenever $Y$ is another Cayley object of $G$ in $\mathcal{K}$, then $X$ and $Y$ are isomorphic if and only if they are isomorphic by a group automorphism of $G$, then we say that $X$ is a CI-object of $G$ in $\mathcal{K}$. If every Cayley object of $G$ in $\mathcal{K}$ is a CI-object of $G$ in $\mathcal{K}$, then we say that $G$ is a CI-group with respect to $\mathcal{K}$. If $G$ is a CI-group with respect to every class of combinatorial objects, then $G$ is a CI-group.

Definition 17. Let $G$ be a finite group. We say that $S \subseteq S_{G}$ is a solving set for a Cayley object $\boldsymbol{X}$ in a class of Cayley objects $\mathcal{K}$ if for every $X^{\prime} \in \mathcal{K}$ such that $X \cong X^{\prime}$, there exists $s \in S$ such that $s(X)=X^{\prime}, s\left(1_{G}\right)=1_{G}$ for every $s \in S$, and $\operatorname{Aut}(G) \leqslant S$. We say that $S \subseteq S_{G}$ is a solving set for a class $\mathcal{K}$ of Cayley objects of $G$ if whenever $X, X^{\prime} \in \mathcal{K}$ are Cayley objects of $G$ and $X \cong X^{\prime}$, then $s(X)=X^{\prime}$ for some $s \in S$, and $s\left(1_{G}\right)=1_{G}$ for every $s \in S$, and $\operatorname{Aut}(G) \leqslant S$. Finally, a set $S$ is a solving set for $\boldsymbol{G}$ if whenever $X, X^{\prime}$ are isomorphic Cayley objects of $G$ in any class $\mathcal{K}$ of combinatorial objects, then $s(X)=X^{\prime}$ for some $s \in S, s\left(1_{G}\right)=1_{G}$ for all $s \in S$, and $\operatorname{Aut}(G) \leqslant S$.

Remark 18. Note that the definition of a solving set given above differs from those in [11] and [3], as here, to simplify both the statements of results and their proofs, we insist that 
every element of the solving set fixes $1_{G}$. It is easy to see that $\alpha^{-1} G_{L} \alpha=G_{L}$ for every $\alpha \in \operatorname{Aut}(G)$, so the image of a Cayley object of $G$ under a group automorphism of $G$ is a Cayley object of $G$. That is, in order to test for isomorphism, automorphisms of $G$ must be considered. However, it is not always the case that every automorphism of $G$ needs to be considered when testing for isomorphism. For example, Cayley graphs of cyclic groups of order $n$ each have an automorphism $x \rightarrow-x$ that is also a group automorphism of $\mathbb{Z}_{n}$, and so the image of a Cayley graph under this automorphism of $\mathbb{Z}_{n}$ is itself. So, while our definition of a solving set is convenient for this paper, it does not always capture the idea behind a solving set (i.e. that it should be as small as possible) exactly, but will only necessarily include extra automorphism of $G$ (which could then be excluded). Also note that in [11] and [3], solving sets were only defined for abelian groups.

Let $X$ be a Cayley object of $G$ in $\mathcal{K}$. We define a CI-extension of $\boldsymbol{G}$ with respect to $\boldsymbol{X}$, denoted by $\mathrm{CI}(G, X)$, to be a set of permutations in $S_{G}$ that each fix $1_{G}$ and whenever $\delta \in S_{G}$ such that $\delta^{-1} G_{L} \delta \leqslant \operatorname{Aut}(X)$, then there exists $v \in \operatorname{Aut}(X)$ such that $v^{-1} \delta^{-1} G_{L} \delta v=t^{-1} G_{L} t$ for some $t \in \mathrm{CI}(G, X)$.

Lemma 19. Let $G$ be a finite group, and $X$ a Cayley object of $G$ in some class $\mathcal{K}$ of combinatorial objects. Then $\mathrm{CI}(G, X)$ exists.

Proof. To show existence, we only need show that there is a set of permutations $T$ in $S_{G}$ such that whenever $\delta \in S_{G}$ such that $\delta^{-1} G_{L} \delta \leqslant \operatorname{Aut}(X)$ and $v \in \operatorname{Aut}(X)$, then $v^{-1} \delta^{-1} G_{L} \delta v=t^{-1} G_{L} t$ for some $t \in T$ and $t\left(1_{G}\right)=1_{G}$. This follows almost immediately. As $\delta v$ is a permutation, there exists $g \in G$ such that $\delta v\left(1_{G}\right)=g$. Let $t_{\delta v}=g_{L}^{-1} \delta v$. Then $t_{\delta v}\left(1_{G}\right)=g_{L}^{-1} \delta v\left(1_{G}\right)=g_{L}^{-1}(g)=g^{-1} g=1_{G}$. Furthermore, $t_{\delta v}^{-1} G_{L} t_{\delta v}=$ $v^{-1} \delta^{-1} g_{L} G_{L} g_{L}^{-1} \delta v=v^{-1} \delta^{-1} G_{L} \delta v$, and existence is established with $T=\left\{t_{\delta v}: \delta^{-1} G_{L} \delta \leqslant\right.$ $\operatorname{Aut}(X), v \in \operatorname{Aut}(X)\}$.

Note for $X$ a Cayley object of $G$ in $\mathcal{K}, \mathrm{CI}(G, X)$ is not unique as if $T$ is CI-extension of $X$ with respect to $G$, then for $\alpha \in \operatorname{Aut}(G),\{\alpha t: t \in T\}$ is also a CI-extension of $X$ with respect to $G$. The following result shows the importance of $\mathrm{CI}(G, X)$, as if $\mathrm{CI}(G, X)$ is known, then the isomorphism problem is solved.

Lemma 20. Let $G$ be a finite group, and $X$ a Cayley object of $G$ in some class $\mathcal{K}$ of combinatorial objects. Then the following are equivalent:

1. $S=\{\alpha t: \alpha \in \operatorname{Aut}(G), t \in T\}$ is a solving set for $X$,

2. $T$ is a $\mathrm{CI}(G, X)$.

Proof. 1) implies 2). Let $\delta \in S_{G}$ such that $\delta^{-1} G_{L} \delta \leqslant \operatorname{Aut}(X)$. Then $\delta(X)$ is a Cayley object of $G$ in $\mathcal{K}$ as $\operatorname{Aut}(\delta(X))=\delta \operatorname{Aut}(X) \delta^{-1} \geqslant G_{L}$. As $S$ is a solving set for $X$, $\delta(X)=s(X)$ for some $s \in S$, and $s=\alpha t$ for some $\alpha \in \operatorname{Aut}(G)$ and $t \in T$. Thus $v=\delta^{-1} s \in \operatorname{Aut}(X)$. Then

$$
\begin{aligned}
v^{-1} \delta^{-1} G_{L} \delta v & =s^{-1} \delta \delta^{-1} G_{L} \delta \delta^{-1} s \\
& =s^{-1} G_{L} s=t^{-1} \alpha^{-1} G_{L} \alpha t \\
& =t^{-1} G_{L} t
\end{aligned}
$$


and $T$ is a $\mathrm{CI}(X, G)$.

2) implies 1). Let $X$ and $X^{\prime}$ be isomorphic Cayley objects of $G$ in $\mathcal{K}$. Then there exists $\delta \in S_{G}$ such that $\delta(X)=X^{\prime}$. As $G_{L} \leqslant \operatorname{Aut}\left(X^{\prime}\right), \delta^{-1} G_{L} \delta \leqslant \operatorname{Aut}(X)$. As $T$ is a $\mathrm{CI}(X, G)$, there exists $t \in T$ and $v \in \operatorname{Aut}(X)$ such that $v^{-1} \delta^{-1} G_{L} \delta v=t^{-1} G_{L} t$. Hence $t v^{-1} \delta^{-1} G_{L} \delta v t^{-1}=G_{L}$. As $G_{L}$ is transitive, there exists $h \in G$ such that $h_{L} \delta v t^{-1}\left(1_{G}\right)=$ $1_{G}$. Clearly then $t v^{-1} \delta^{-1} h_{L}^{-1} G_{L} h_{L} \delta v t^{-1}=G_{L}$ so that $h_{L} \delta v t^{-1}$ normalizes $G_{L}$ and fixes $1_{G}$. By [2, Corollary 4.2B], we have that $h_{L} \delta v t^{-1}=\alpha \in \operatorname{Aut}(G)$. Then $\alpha t v^{-1} \delta^{-1} h_{L}^{-1}=\left(1_{G}\right)_{L}$ and $\alpha t=h_{L} \delta v$. Then $\alpha t(X)=h_{L} \delta v(X)=h_{L} \delta(X)=h_{L}\left(X^{\prime}\right)=X^{\prime}$.

The following result shows that if a solving set for $X$ has been found, then some $\mathrm{CI}(G, X)$ has also been found.

Lemma 21. Let $G$ be a group, $X$ a Cayley object of $G$, and $S$ a solving set for $X$. Define an equivalence relation $\equiv$ on $S$ by $s_{1} \equiv s_{2}$ if and only if $s_{1}=\alpha s_{2}$ for some $\alpha \in \operatorname{Aut}(G)$. Let $T$ be a set consisting of one representative from each equivalence class of $\equiv$. Then $T$ is a $\mathrm{CI}(G, X)$.

Proof. It is straightforward to show that $\equiv$ is indeed an equivalence relation. Choose a $T$ as is given in the statement. Let $X^{\prime}$ be a Cayley object of $G$ isomorphic to $X$ with $\delta: X \rightarrow X^{\prime}$ an isomorphism. Then $\delta^{-1} G_{L} \delta \leqslant \operatorname{Aut}(X)$. Also, as $S$ is a solving set for $X$, there exists $s \in S$ such that $s(X)=X^{\prime}$ so that $v=\delta^{-1} s \in \operatorname{Aut}(X)$. Let $t \in T$ such that $t \equiv s$ so that $\alpha t=s$ for some $\alpha \in \operatorname{Aut}(G)$. Then $v^{-1} \delta^{-1} G_{L} \delta v=t^{-1} \alpha^{-1} G_{L} \alpha t=$ $t^{-1} G_{L} t$.

Let $\mathcal{K}$ be a class of combinatorial objects, and $G$ a group. We define a CI-extension of $G$ with respect to $\mathcal{K}$, denoted by $\mathrm{CI}(G, \mathcal{K})$, to be a set of permutations in $S_{G}$ that each fix $1_{G}$ and whenever $X \in \mathcal{K}$ is a Cayley object of $G$ and $\delta \in S_{G}$ such that $\delta^{-1} G_{L} \delta \leqslant$ $\operatorname{Aut}(X)$, then there exists $t \in \mathrm{CI}(G, \mathcal{K})$ and $v \in \operatorname{Aut}(X)$ such that $v^{-1} \delta^{-1} G_{L} \delta v=t^{-1} G_{L} t$. The proofs of the following results are straightforward.

Lemma 22. Let $G$ be a finite group, and $\mathcal{K}$ a class of combinatorial objects. Then the following are equivalent:

1. $S=\{\alpha t: \alpha \in \operatorname{Aut}(G), t \in T\}$ is a solving set for $G$ in $\mathcal{K}$,

2. $T$ is a $\mathrm{CI}(G, \mathcal{K})$.

Lemma 23. Let $G$ be a group, $\mathcal{K}$ a class of combinatorial objects, and $S$ a solving set for $G$ in $\mathcal{K}$. Define an equivalence relation $\equiv$ on $S$ by $s_{1} \equiv s_{2}$ if and only if $s_{1}=\alpha s_{2}$ for some $\alpha \in \operatorname{Aut}(G)$. Let $T$ be a set consisting of one representative from each equivalence class of $\equiv$. Then $T$ is a $\mathrm{CI}(G, \mathcal{K})$.

Let $G$ be a finite group. We define a CI-extension of $\boldsymbol{G}$, denoted by $\mathrm{CI}(G)$, to be a set of permutations in $S_{G}$ that each fix $1_{G}$ and whenever $X \in \mathcal{K}$ is a Cayley object of $G$ in some class $\mathcal{K}$ of combinatorial objects, and $\delta \in S_{G}$ such that $\delta^{-1} G_{L} \delta \leqslant \operatorname{Aut}(X)$, then there exists $t \in \mathrm{CI}(G)$ and $v \in\left\langle G_{L}, \delta^{-1} G_{L} \delta\right\rangle$ such that $v^{-1} \delta^{-1} G_{L} \delta v=t^{-1} G_{L} t$. 
Repeated application of Lemma 19 for every combinatorial object $X$ in every class $\mathcal{K}$ of combinatorial objects shows that $\mathrm{CI}(G)$ exists. The proofs of the following results are straightforward.

Lemma 24. Let $G$ be a finite group. Then the following are equivalent:

1. $S=\{\alpha t: \alpha \in \operatorname{Aut}(G), t \in T\}$ is a solving set for $G$,

2. $T$ is a $\mathrm{CI}(G)$.

Lemma 25. Let $G$ be a group, and $S$ a solving set for $X$. Define an equivalence relation $\equiv$ on $S$ by $s_{1} \equiv s_{2}$ if and only if $s_{1}=\alpha s_{2}$ for some $\alpha \in \operatorname{Aut}(G)$. Let $T$ be a set consisting of one representative from each equivalence class of $\equiv$. Then $T$ is a $\mathrm{CI}(G)$.

\section{Applications}

At the present time, the isomorphism problem has not been solved for any nilpotent group that is not abelian in any class of combinatorial objects, so there are not at this time any applications for Theorem 14 (1) (although as soon as the isomorphism problem has been solved for any nonabelian $p$-group in certain classes of combinatorial objects, such as color digraphs, that will change immediately). We do though have an application of Theorem 14 (2) which will not only provide new examples of CI-groups with respect to color digraphs, but also illustrate how Theorem 14 (2) generalizes the main result of [3].

The following result weakens the hypothesis (replacing normally $s$-step imprimitive with $s$-step imprimitive) of [3, Theorem 16] and generalizes this result from abelian to nilpotent groups.

Theorem 26. Let $n_{1}, \ldots, n_{r}$ be positive integers such that $\operatorname{gcd}\left(n_{i}, n_{j} \cdot \varphi\left(n_{j}\right)\right)=1$ if $i \neq j$, and $\pi_{i}$ be the set of distinct prime numbers dividing $n_{i}$. Let $n=n_{1} \cdots n_{r}, \Omega(n)=m$, and $G$ a nilpotent group of degree $n$. Let $G=\prod_{i=1}^{r} N_{i}$ where each $N_{i}$ is a $\pi_{i}$-subgroup of $G$, and $S(i)$ a solving set for $N_{i}$. If

1. each $n_{i}$ is prime-power or $G$ is abelian, and

2. whenever $\delta \in S_{G}$ there exists $\phi \in\left\langle G_{L}, \delta^{-1} G_{L} \delta\right\rangle$ such that $\left\langle G_{L}, \phi^{-1} \delta^{-1} G_{L} \phi \delta\right\rangle$ is m-step imprimitive,

then $\prod_{i=1}^{r} S(i)$ is a solving set for $G$.

Proof. Let $\delta \in S_{G}$. By the hypothesis, we have that there exists $\phi \in\left\langle G_{L}, \delta^{-1} G_{L} \delta\right\rangle$ such that $\left\langle G_{L}, \phi^{-1} \delta^{-1} G_{L} \delta \phi\right\rangle$ is $m$-step imprimitive. By Theorem 14, there exists $\omega \in$ $\left\langle G_{L}, \phi^{-1} \delta^{-1} G_{L} \delta \phi\right\rangle$ such that $L=\left\langle G_{L}, \omega^{-1} \phi^{-1} \delta^{-1} G_{L} \delta \phi \omega\right\rangle=\Pi_{i=1}^{r} L_{i}$, where each $L_{i}$ is a transitive $\pi_{i}$-group of degree $n_{i}, 1 \leqslant i \leqslant r$. Let $\mathrm{CI}\left(N_{i}\right)$ be a CI-extension of $N_{i}$ as given by Lemma 25. As $S(i)$ is a solving set for $N_{i}$, by Lemma 24 there exists $t_{i} \in \mathrm{CI}\left(N_{i}\right)$ and $v_{i} \in L_{i}$ such that $v_{i}^{-1}\left(\left(\omega^{-1} \phi^{-1} \delta^{-1} G_{L} \delta \phi \omega\right) / \mathcal{C}_{i}\right) v_{i}=t_{i}^{-1}\left(G_{L} / \mathcal{C}_{i}\right) t_{i}$, where $\mathcal{C}_{i}$ is the complete block system of $L$ formed by the orbits of $\prod_{j=1, j \neq i}^{r} L_{i}$. Let $t=\left(t_{1}, \ldots, t_{r}\right)$ 
and $v=\left(v_{1}, \ldots, v_{r}\right)$. Note that $t$ fixes $1_{G}$. As $L=\prod_{i=1}^{r} L_{i}$, we have that $v \in L$ and $t^{-1} G_{L} t=v^{-1} \omega^{-1} \phi^{-1} \delta^{-1} G_{L} \delta \phi \omega v$. By definition, $\Pi_{i=1}^{r} \mathrm{CI}\left(N_{i}\right)$ is a CI-extension of $G$, and so by Lemma 24, $S=\left\{\alpha t: t \in \Pi_{i=1}^{r} \mathrm{CI}\left(N_{i}\right), \alpha \in \operatorname{Aut}(G)\right\}$ is a solving set for $G$. Finally, it is not difficult to see that $\operatorname{Aut}(G)=\prod_{i=1}^{r} \operatorname{Aut}\left(N_{i}\right)$ as $G$ is nilpotent and if $i \neq j$ then $\operatorname{gcd}\left(n_{i}, n_{j}\right)=1$, and so $\alpha=\left(\alpha_{1}, \alpha_{2}, \ldots, \alpha_{r}\right), \alpha_{i} \in \operatorname{Aut}\left(N_{i}\right)$. Then $\alpha t=\left(\alpha_{1} t_{1}, \alpha_{2} t_{2}, \ldots, \alpha_{r} t_{r}\right), \alpha_{i} \in \operatorname{Aut}\left(N_{i}\right)$ and $t_{i} \in \mathrm{CI}\left(N_{i}\right)$. Thus $\alpha t \in \prod_{i=1}^{r} S(i)$, $S \leqslant \prod_{i=1}^{r} S(i)$, and $\prod_{i=1}^{r} S(i)$ is a solving set for $G$.

Definition 27. Let $\Omega$ be a set. A $\boldsymbol{k}$-ary relational structure on $\Omega$ is an ordered pair $(\Omega, U)$, where $U \subseteq \Omega^{k}=\prod_{i=1}^{k} \Omega$. A group $G \leqslant S_{\Omega}$ is called $\boldsymbol{k}$-closed if $G$ is the intersection of the automorphism groups of some set of $k$-ary relational structures. The $\boldsymbol{k}$-closure of $\boldsymbol{G}$, denoted $G^{(k)}$, is the intersection of all $k$-closed subgroups of $S_{\Omega}$ that contain $G$.

Note that a 2-closed group is the automorphism group of a color digraph. The following result of Kalužnin and Klin [7] will prove useful.

Lemma 28. Let $G \leqslant S_{X}$ and $H \leqslant S_{Y}$. Let $G \times H$ act canonically on $X \times Y$. Then $(G \times H)^{(k)}=G^{(k)} \times H^{(k)}$ for every $k \geqslant 2$.

If, in Theorem $26, \mathcal{K}$ is the class of $k$-ary relational structures, and the groups $L / \mathcal{C}_{i}$ are as in the proof of Theorem 26, then by Lemma 28 we may assume that each $L / \mathcal{C}_{i}$ is $k$-closed (although there is no reason to believe that each $L / \mathcal{C}_{i}$ is a $\pi_{i}$-subgroup - but this fact is not used in the proof of Theorem 26). Proceeding as in Theorem 26 and applying Lemma 22 instead of Lemma 24, we have the following result.

Corollary 29. Let $n_{1}, \ldots, n_{r}$ be positive integers such that $\operatorname{gcd}\left(n_{i}, n_{j} \cdot \varphi\left(n_{j}\right)\right)=1$ if $i \neq j$, and $\pi_{i}$ be the set of distinct prime numbers dividing $n_{i}$. Let $n=n_{1} \cdots n_{r}, \Omega(n)=m$, and $G$ a nilpotent group of degree $n$. Let $G=\prod_{i=1}^{r} N_{i}$ where each $N_{i}$ is a $\pi_{i}$-subgroup of $G$, and $S(i)$ a solving set for $N_{i}$ in the class of $k$-ary relational structures. If

1. each $n_{i}$ is prime or $G$ is abelian, and

2. whenever $\delta \in S_{G}$ there exists $\phi \in\left\langle G_{L}, \delta^{-1} G_{L} \delta\right\rangle$ such that $\left\langle G_{L}, \phi^{-1} \delta^{-1} G_{L} \phi \delta\right\rangle$ is m-step imprimitive,

then $\prod_{i=1}^{r} S(i)$ is a solving set for $G$ in the class of $k$-ary relational structures.

We now give the promised application of Theorem 14 (2) which gives new CI-groups with respect to Cayley color digraphs of a particular group. Using the results in [3], this result could be obtained but only in the special cases where the following additional arithmetic conditions hold: $p$ does not divide $q-1$, and each $n_{i}$ is prime. Before proceeding, we need a preliminary lemma.

Lemma 30. Let $n$ be a positive integer with a prime divisor $p \mid n$ such that $n / p<p$. If $G$ is a regular group of order $n$, and $\phi \in S_{n}$, then there exists $\delta \in\left\langle G, \phi^{-1} G \phi\right\rangle$ such that $\left\langle G, \delta^{-1} \phi^{-1} G \phi \delta\right\rangle$ admits a normal complete block system with blocks of size $p$. 
Proof. First observe that as $n / p<p, G$ must have a unique cyclic Sylow $p$-subgroup $P$ of order $p$. So $P \triangleleft G$ and $G$ admits a normal complete block system $\mathcal{B}$ consisting of blocks of size $p$, formed by the orbits of $P$. Furthermore, a Sylow $p$-subgroup of $S_{n}$ is of the form $1_{S_{n / p}}\left\{\mathbb{Z}_{p}\right.$, so we see that $\left\langle\left. P\right|_{B}: B \in \mathcal{B}\right\rangle$ is a Sylow $p$-subgroup of $S_{n}$. By a Sylow theorem, there exists $\delta \in\left\langle G, \phi^{-1} G \phi\right\rangle$ such that $\delta^{-1} \phi^{-1} G \phi \delta \leqslant\left\langle\left. P\right|_{B}: B \in \mathcal{B}\right\rangle$. Let $P_{1}$ be the largest subgroup of $\left\langle G, \delta^{-1} \phi^{-1} G \phi \delta\right\rangle$ contained in $\left\langle\left. P\right|_{B}: B \in \mathcal{B}\right\rangle$. Then $G$ normalizes $P_{1}$ as does $\delta^{-1} \phi^{-1} G \phi \delta$. Hence the orbits of $P_{1}$, which is $\mathcal{B}$, is a complete block system of $\left\langle G, \delta^{-1} \phi^{-1} G \phi \delta\right\rangle$ and the result follows.

Theorem 31. Let $p$ and $q$ be distinct primes with $p^{2}<q$, and $q_{1}, \ldots, q_{r}$ distinct primes such that $q p^{2}<q_{1}$ and $q p^{2} q_{1} \cdots q_{i}<q_{i+1}, 1 \leqslant i \leqslant r-1$. Let $m=q_{1} \cdots q_{r}, n_{0}=p^{2} q$, and $n_{1}, \ldots, n_{s}$ divisors of $m$ such that $n_{1} \cdots n_{s}=m$. If $\operatorname{gcd}\left(n_{i}, n_{j} \cdot \varphi\left(n_{j}\right)\right)=1$ then $\mathbb{Z}_{q} \times \mathbb{Z}_{p}^{2} \times \mathbb{Z}_{m}$ is a CI-group with respect to digraphs.

Proof. Let $\Gamma$ be a Cayley color digraph of $G=\mathbb{Z}_{q} \times \mathbb{Z}_{p}^{2} \times \mathbb{Z}_{m}$, and $\phi \in S_{G}$ such that $\phi^{-1} G_{L} \phi \leqslant \operatorname{Aut}(\Gamma)$. We first show by induction on $r$ that $\left\langle G_{L}, \phi^{-1} G_{L} \phi\right\rangle$ is $s$-step imprimitive, where $s=r+3$. If $r=0$, then as $p^{2}<q$ by Lemma 30 we may assume after an appropriate conjugation that $\left\langle G, \phi^{-1} G \phi\right\rangle$ admits a (normal) complete block system $\mathcal{B}_{q}$ with blocks of size $q$. Then $G_{L} / \mathcal{B}_{q}$ and $\phi^{-1} G_{L} \phi / \mathcal{B}_{q}$ are contained in conjugate Sylow $p$-subgroups of $\left\langle G_{L}, \phi^{-1} G_{L} \phi\right\rangle / \mathcal{B}_{q}$, and so after another appropriate conjugation we may assume that $\left\langle G_{L}, \phi^{-1} G_{L} \phi\right\rangle / \mathcal{B}_{q}$ is a $p$-group. The center of $\left\langle G_{L}, \phi^{-1} G_{L} \phi\right\rangle / \mathcal{B}_{q}$ contains an element of order $p$ whose orbits give a complete block system $\mathcal{B}_{p}$ consisting of blocks of size $p$. Then $\mathcal{B}_{p}$ induces a complete block system $\mathcal{B}_{q} \prec \mathcal{B}_{p q}$ of $\left\langle G_{L}, \phi^{-1} G_{L} \phi\right\rangle$ with blocks of size $p q$. Hence $\left\langle G_{L}, \phi^{-1} G_{L} \phi\right\rangle$ is 3-step imprimitive. Assume that $\left\langle G_{L}, \phi^{-1} G_{L} \phi\right\rangle$ is sstep imprimitive when $r \geqslant 0$ and let $G_{L}$ be such that $m$ is a product of $r+1$ primes. Again, after an application of Lemma 30, we may assume that $\left\langle G_{L}, \phi^{-1} G_{L} \phi\right\rangle$ admits a complete block system $\mathcal{B}_{r+1}$ with blocks of size $q_{r+1}$. By induction we may assume that $\left\langle G_{L}, \phi^{-1} G_{L} \phi\right\rangle / \mathcal{B}_{q_{r+1}}$ is $(r+3)$-step imprimitive. It is then not difficult to see that $\left\langle G_{L}, \phi^{-1} G_{L} \phi\right\rangle$ is $(r+4)$-step imprimitive. By induction, we then have $\left\langle G_{L}, \phi^{-1} G_{L} \phi\right\rangle$ is $(r+3)$-step imprimitive as required.

Write $G_{L}=\prod_{i=0}^{s} G_{i}$, where $G_{i} \leqslant G_{L}$ is of order $n_{i}$. As $G_{i}$ is a CI-group with respect to Cayley color digraphs by [8] if $i=0$ and by [10] otherwise, by Corollary 29, we see that if $S$ is a solving set of $G$ in the class of color digraphs then $S \subseteq \prod_{i=1}^{r} \operatorname{Aut}\left(G_{i}\right) \leqslant \operatorname{Aut}(G)$. Hence $G$ is a CI-group with respect to color digraphs.

\section{References}

[1] A. Ádám. Research problem 2-10. J. Combin. Theory 2:393, 1967.

[2] John D. Dixon and Brian Mortimer. Permutation groups. Graduate Texts in Mathematics, vol. 163, Springer-Verlag, New York, 1996.

[3] Edward Dobson. On isomorphisms of abelian Cayley objects of certain orders. Discrete Math. 266:203-215, 2003. 
[4] Bernard Elspas and James Turner. Graphs with circulant adjacency matrices J. Combinatorial Theory 9:297-307, 1970.

[5] Daniel Gorenstein. Finite groups. Harper \& Row Publishers, New York, 1968.

[6] Marshall Hall, Jr. The theory of groups. Chelsea Publishing Co., New York, 1976, Reprinting of the 1968 edition.

[7] L. A. Kalužnin and M. H. Klin. Some numerical invariants of permutation groups. Latviǔsk. Mat. Ežegodnik 18:81-99, 1976.

[8] I. Kovács and M. Muzychuk. The group $\mathbb{Z}_{p}^{2} \times \mathbb{Z}_{q}$ is a CI-group. Comm. Algebra 37:3500-3515, 2009.

[9] J. D. P. Meldrum. Wreath products of groups and semigroups. Pitman Monographs and Surveys in Pure and Applied Mathematics, vol. 74, Longman, Harlow, 1995.

[10] Mikhail Muzychuk. On Ádám's conjecture for circulant graphs Discrete Math. 176:285-298, 1997.

[11] Mikhail Muzychuk. On the isomorphism problem for cyclic combinatorial objects. Discrete Math. 197/198:589-606.

[12] M. Muzychuk and F. Pakovich. Jordan-Hölder theorem for imprimitivity systems and maximal decompositions of rational functions. Proc. Lond. Math. Soc. 102:1-24, 2011.

[13] P. P. Pálfy. Isomorphism problem for relational structures with a cyclic automorphism. European J. Combin. 8:35-43, 1987.

[14] Helmut Wielandt, Finite permutation groups. Translated from the German by R. Bercov, Academic Press, New York, 1964. 